\title{
Approaches for the Synthesis of Decision-Makings Review
}

\author{
Denis P. Oleynikov \\ CAD/CAE Systems department \\ Volgograd State Technical University \\ Volgograd, Russia \\ denis.oleynikov@gmail.com
}

\author{
Lyudmila N. Butenko \\ CAD/CAE Systems department \\ Volgograd State Technical University \\ Volgograd, Russia \\ butenko@vstu.ru
}

\begin{abstract}
This paper describes author's approaches, intended to obtain new decision-making methods. Approaches reflect morphological aspects of the consideration of decisionmakings affect system-wide trends of the development of systems and contain elements of the heuristic nature of the synthesis process. Common for these approaches is the consideration of the synthesis of objects as systems with the goal structure and properties.
\end{abstract}

Keywords: decision-makings, system synthesis, morphological synthesis, heuristical synthesis

\section{INTRODUCTION}

The need of creation of new decision-makings can be caused by the decision of the new, or improve the efficiency of the existing complex and poorly formalized tasks. Some of these problems are:

- Problems solved within the framework of the doctrine of network-centric warfare to enhance the combat capabilities of modern formations [1].

- Automation and control of modern nuclear power plants. One of the main trends of development of NPP ACS is currently a growing volume of processed information, conditioned by the more widespread use of "smart" sensors, and the natural desire of operating personnel "to increase the zone (area) monitoring" of the process [2].

- The decision of forecasting problems with different time horizons [3]. Solving problems of choice of appropriate forecasting models and methods as a result of the increasing complexity of these methods and models [4]. At the same time, forecasting models and methods can be used to form a strategy for the development of technology and innovation.

- The decision of creative tasks, which associated with the conceptual design of complex objects in science, engineering and education [5].

Parameters of subject area can significantly limit the set of admissible decision-makings, up to the empty set [6]. For this reason, the problem of the synthesis of new decision-makings, that will effectively solve the tasks of choosing alternatives in areas, where the application of existing methods is unreasonable and unacceptable, is actual [7].
To solve this problem the authors have developed a number of approaches to the synthesis of the decision-making methods that are listed in the next sections.

\section{APPROCHES FOR THE SYNTHESIS OF DECISION- MACKINGS}

\section{A. Approach of synthesis based on polarity principle}

This principle expresses the idea of the duality of any objects of nature as a fundamental property of the real world. Development of the object is the result of synthesis of two opposing principles. The approach consists of the following steps [8]:

1) Determine the synthesis goal.

2) Select a pair of systems as base of the synthesis of the new system, a set of characteristics corresponding to the goal. It is advisable to pre-analyze the basic systems with indicating of main structural elements and functions.

3) Make a comparative analysis systems (subsystems) with bipolar scales of attributes. At this stage it is necessary to allocate a number of useful properties that correspond to the characteristics of systems (subsystems), included in the base of the sysnthesis.

4) Select the basic subsystem, upon which the new system is synthesized. The base subsystem is most full corresponds to the synthesis of goal;

5) Formulate requirements for the system (subsystem) to be created using selected strategy (goals) and limitations imposed by the previously synthesized systems (subsystems);

6) Select the elements of basic systems that implement requirements;

7) For each element establish conditions for the using in the new system;

8) Transform selected elements, or add new elements of external systems to resolve the conflicting demands of adjacent elements.

9) Compose subsystems considering formulated requirements from the modified elements of compared systems, and from elements of external systems that satisfy formulated requirements.

10) Perform repeated synthesis (if necessary) of the previously created systems (subsystems); 
11) Compose goal system considering formulated requirements from the modified elements of compared systems, and from elements of external systems that satisfy formulated requirements.

At various stages of the synthesis approach, each of decisionmakings included in base synthesis, considered as a system consisting of various subsystems. An example of using this approach is given in [9], where described a process of synthesis of the verbal hierarchical classification method. An example of using this approach for synthesis of ARACE verbal decision making is method given in $[10,11]$.

\section{B. Approach of synthesis of object-and problem-oriented decision-makings based on morpological principle}

This approach is based on the morphological method Zwicky and uses heuristics to improve the functions of decision-making to be created [12]. A significant advantage of the morphological method Zwicky is its relatively easy to implement by hardware. This helps to reduce the time costs in forming of the initial set of variants of the solution. The approach consists two stages - the goal setting (GS) and the goal achievement (GA). In general terms, goal setting - the primary control phase of the synthesis, which provides a statement of the general purpose and set of goals (objectives tree) in accordance with the purpose of the system, strategic settings and the nature of tasks. Goal achievement - the active control synthesis phase, which consists of transition of the object of synthesis from the current state to the target state.

\section{Goal setting stage consists of following steps:}

1) The analysis of the decision-making, which consists of determining the composition of functions, the algorithm of the method, advantages and disadvantages, the boundaries of their applicability.

2) The definition of classification criteria of decisionmaking.

3) The determination development trends of decisionmaking.

4) The classification of decision-making.

5) The formalization of development trends of decisionmaking.

6) The determination of the classification criteria of problems of synthesis problem - and object-oriented methods.

7) The inversion of part of classification criteria for the production the synthesis problem.

8) The formulation of the synthesis problem.

9) The construction of the mask of the synthesis. The mask is divided into two kinds of requirements: "hard" and "soft" requirements, which reduce the set of possible solutions.

10) The imposition of the synthesis mask on a set of methods for obtaining basic set of decision-making, that will be involved in the synthesis process.

11) The decomposition of decision-making into subsystems, making description of subsystems, analysis of information flows in and between of subsystems.

12) The definition of hierarchy of functions and classification criteria for description in a synthesized decision-making, determine the types of parametersof subsystems.

13) The construction of the table of candidate morphological subsystems involved in the synthesis process.

The goal achievement stage consists of following steps:

1) Evaluate candidate subsystems and construct their models;

2) Synthesis of new subsystems method which comprises the following steps:

- Construction of models of candidate subsystems.

- Consideration of information flows in candidate subsystems.

- Construction of the generalized model of the synthesized method.

- Analysis of models of candidate subsystems for compliance with the generalized model of the synthesized method.

- Resolving the possible consistency problem of new decision-making by using of the following approaches: localization of incompatibility; reduction of incompatible elements to the particular case; exclusion requirements.

3) The development of functionality of synthesized method with known approaches.

The division into stages of goal setting and goal achievement in the synthesis is conventional and does not have clear boundaries. Step goal-setting stage is completed when the task of synthesis is defined. However, since the problem is constantly refined, this stage lasts longer and does not stop at once. The same is true about the goal achievement stage.

This approach has been used to create a DSS Unicum [13], intended for ranking alternatives that have both qualitative and quantitative criteria estimates.

\section{Approach of synthesis based on morpological principle and three-stage matching}

To use this approach, the way describing of decisionmakings was identified. In this way each method is considered as a set of sequential steps (processes) transformation of information until the initial solution have received [14]. The ADSM- model (Attribute, Data-flow, Step, Method) is used for description of decision-makings:

- $\quad a$ - the attribute is a tuple $\langle$ aName, aGroup $\rangle ;$ aNamename of the attribute; aGroup - group of the attribute.

- $d$ - the data-flow is a tuple $\langle d N a m e, A\rangle ;$ dName name of the data-flow; $A=\left\{a_{1}, \ldots, a_{d}\right\}-$ set of attributes of the data-flow.

- $s$ - the step is a tuple $\left\langle s\right.$ Name, $\left.D_{\text {in }}, D_{\text {out }}, A\right\rangle$; sName - name of the step; $D_{\text {in }}=\left\{d_{1}, \ldots, d_{m}\right\}$ - set 
of input data-flows, where $m$ - count of input dataflows of the step; $D_{\text {out }}=\left\{d_{1}, \ldots, d_{n}\right\}$ - set of output data-flows, where $n$-count of output data-flows of the step; $A=\left\{a_{1}, \ldots, a_{s}\right\}$ - set of attributes of the step of the decision-making.

- $\mathrm{m}$ - the decision-making is an ordered set of the steps $\left\langle s_{1}, \ldots, s_{k}\right\rangle$, which transforms input data-flows into output data-flows.

For structured elements were defined next operations:

- $D_{\text {in }}(s)=\left\{d_{1}, \ldots, d_{n}\right\}$ - getting the set of input data-flows of the $\operatorname{step}^{s}$.

- $D_{\text {out }}(s)=\left\{d_{1}, \ldots, d_{m}\right\}$ - getting the set of output dataflows of the step $s$.

- $A(s)=\left\{a_{1}, \ldots, a_{k}\right\} \mathrm{A}(\mathrm{s})=\left\{\mathrm{a}_{1}, \mathrm{a}_{2}, \ldots, \mathrm{a}_{\mathrm{k}}\right\}-$ getting the set of attributes of the step ${ }^{s}$.

- $A(d)=\left\{a_{1}, \ldots, a_{t}\right\} \mathrm{A}(\mathrm{d})=\left\{\mathrm{a}_{1}, \mathrm{a}_{2}, \ldots, \mathrm{a}_{\mathrm{t}}\right\}-$ getting the set of attributes of the data-flow $d$. - $\quad \operatorname{Group}(a)=a$ Group - getting the group of the attribute

The prerequisites of the synthesis procedure are:

1) All decision-makings must be decomposed into sequential steps and must be described as information objects.

2) "Given" and "required" data-flows must be defined.

When all prerequisites were performed, the block-labyrinth morphological method performs iteratively. The condition of existence of the solution is: at least one output data-flow of the previous step corresponds with one incoming data-flow of the next step. The determinacy of the step calculates during synthesis process. The determinacy is ratio of the number of linked input data-flows related to the total number of input dataflows of the step. Then solutions are sorted by the multiplicative criterion and selected best ones.

The essence of the solution is the conceptual structure the conversion process of input data into the output data.

This approach was implemented in the synthesis support system of decision-makings "Alpha-Synthesis" [15], by which a number of decision-makings were synthesized.

\section{Approach of synthesis based on heuristic patterns}

To create this approach the group of criteria of game theory with the nature has been analyzed. The recurrence of some of the structural elements in different criteria was detected in the results of the comparative analysis, where ontology schemas were built. These repetitive elements formed the basis for the creation of structural patterns database. Below are examples of some patterns:
1) Variability of integral estimation. The estimate is form using an integral summation, product, determining the average value, etc., which is then use to make a decision.

2) Filter. The decision depends on the parameter of the system, which does not participate in the formation of integral estimate of alternatives. The parameter filters out unwanted options of the solution, amd may be an external parameter, or formed in the process of decision making.

3) Regulator. The element, which regulates the degree of realization of each of the inverse (in the general case different) functions, integrates into the decision-making Functions are executed in parallel. It is possible to use two or more functions.

4) Hierarchy. Decision making is carried out in several stages, between which there is approval procedure intermediate solutions, the results of the previous stages are used at the next level of the hierarchy.

In general, the synthesis approach with the use of heuristic patterns consists of the following steps:

1) The multidimensional analysis of the decision-makings. Determination of structural and functional elements.

2) Construction of models analyzed the decision-makings.

3) Setting the goal of synthesis..

4) Formation of base of synthesis - a set of decisionmakings, which collection of properties meet the synthesis goal.

5) Selection of the prototype method.

6) Convert the structural scheme of the method-prototype using the heuristic patterns, which corresponds with the synthesis goal, and complement it with the necessary subsystems of decision-making, included in the synthesis of the base.

7) Matching the target decision-making with heuristic patterns, designed to solve matching problems.

8) Evaluation of the decision-making on the subject of the expansion limits of applicability, the account large number of environmental factors, the accuracy of decision-making and other specified performance indicators.

Examples of using of this approach are given in [16], where the inversion of forming of estimate of alternative heuristic pattern was used.

\section{THE RESUltS OF ANALISYS OF APPROACHES}

The analysis of the sequence of operations performed by designer to create of new decision-makings, allowed to identify main stages of the system synthesis process of decision-making: selection the method of synthesis, where the target state decision-making is formed; formation of base of synthesis, where the set of elements which will compose the target method of decision-making is selected; combination of elements and agreeing target method of decision-making and its subsystems, where the conceptual structure of the target method is formed; variation, where the family of new methods is created with using if intermediate results of the synthesis. In general, the steps can be divided into groups: goal setting (GS), goal achievement (GA) and the variation of the goal (VG). 
The basis of the majority of procedures that are used in the analysis of the approach to the synthesis of the decision-making, are:

1) The inversion, which is used to ensure maximum growth of novelty.

2) The combinatorial approach, wich is used to maximize the result of variation.

3) The formation of a field of knowledge, which is based on the properties or functional / elemental composition of the decision-making.

The problem the synthesis can be described formally as follows: $\mathrm{X}=<\mathrm{F}, \mathrm{A}, \mathrm{F}_{\mathrm{A}}, \mathrm{F}_{\mathrm{B}}, \mathrm{C}, \mathrm{C}_{\mathrm{s}}, \mathrm{C}_{\mathrm{f}}, \mathrm{M}, \mathrm{E}>$, where:

- $\mathrm{F}$ - the set of characteristics, which describes the synthesis problem.

- $\mathrm{A}$ - the decision making environment described by verbal way.

- $\quad \mathrm{F}_{\mathrm{A}}$ - the set of the characteristics of decision making environment, that are restrictions imposed on the synthesis result.

- $\mathrm{F}_{\mathrm{B}}$ - the set of characteristics that should have the target method decision making.

- $\mathrm{C}$ - set of the existing decision-making methods, each of which is presented in the form of structural $C_{s}$ and functional $\mathrm{C}_{\mathrm{f}}$ model.

- $\quad$ The $\mathrm{C}_{\mathrm{s}}$ and $\mathrm{C}_{\mathrm{c}}$ models which are presented as the set of structural and functional elements with defined relationships.

- $\mathrm{M}$ - the compatibility matrix of properties, which determines the possibility of combining the existing structural and functional properties of subsystems.

- E - set of synthesis control rules that determine the variability of the target system, the synthesis depth algorithm for selecting optimal decision on a wave.

The synthesis process can be described as follows:

1) Forming sets $F_{A}$ - the selection of characteristics that may be used to describe the target and decision-making environment.

2) Forming set $C$-definition of the decision-makings that will be the prototype of the target decision-making.

3) Forming models $C_{s}$ and $C_{f}$ - carrying multidimensional analysis of the decision-makings. Selecting and decomposition of functional subsystems and structural elements.

4) The mapping between elements of models $C_{s}$ and $C_{f}$ and set $F$.

5) Forming set $E$-assignment synthesis management rules.

6) Forming set $F_{B}$-definition of synthesis goal.

7) Forming the synthesis base $D-$ a sets of functional, structural elements, which properties match with synthesis conditions
8) Forming variants of the target system from elements of set $D$ with taking into account of defined requirements $F_{A}, F_{B}$.

9) Evaluation of obtained results with rules $E$ and transition to the next iteration of synthesis or stop synthesis process.

The novelty of the result can be estimated using the approach in which result characteristics compared with models and specifications $\mathrm{C}_{\mathrm{S}} \mathrm{C}_{\mathrm{f}}$ of prototype-methods.

Target system quality indicator is an emergent property, which can be defined by expert as the degree of achieve the stated targets.

In accordance with the results of analysis the following conclusions can be made:

1) The target component of the choice of different approaches to the synthesis of decision-akings is information about the decision making environment, decision-makings which can be selected as prototypes and the degree of their formalization.

2) Heuristic approaches can be selected in a situation where there is a lack of objective information about the environment and prototypes of decision-makings, and the main operations of the synthesis are performed by constructor.

\section{CONCLUSION}

The result of choice of approach to the synthesis depends on the result of the multi-dimensional analysis of the decisionmakings.

Heuristic approaches are primarily designed to reduce the dimension of the feasible set of solutions, reducing the burden on the designer, on the process of the execution of cognitive operations and can be used in non-formalized statement of the problem in the synthesis.

Morphological approach should be used in situations where there is a description of the purpose and decision- makings as a set of characteristic features and it is possible to build morphological matrix. In this case, the efficiency of the process of synthesis can be improved by automating the generation variants of solution.

The goal setting stage is crucial in the sequence of steps of synthesis of new system. The goal can be set in advance or may be formed as a result of the analysis of the problem area. The purpose of the synthesis may be: the expansion of the range of applicability of the existing decision-makings, the elimination of the existing shortcomings, the adaptation to the new conditions of the decision making environment. The input data for the group stages of goal setting is a description of the problem.

In this context, becomes relevant automation tasks, which currently can be solved with the help of designer cognitive operations only:

1) Selection and ranking functions to be forcing of.

2) Identification and ranking of the shortcomings of the decision-makings in the case of inversion.

3) Identification of problem areasand their formalization and ranking. 
4) The choice of the most appropriate development trends, taking into account of which leads to creation of new efficient decision-makings.

The goal achievement stage actualizes goal, formed at the goal setting stage. As well as goal setting stage, the goal achievement stage performed at various levels of decisionmaking during the synthesis process.

The goal achievement stage taking into account goal of synthesis generates decision-making. Moreover, the formation of the synthesis base, arrangement, eliminates conflicts between components (if necessary, re-synthesis of components) are performed for the target system and each of their subsystems.

Upon completion of the synthesis process, the process of variations of the target decision-making is running. In this process, the obtained decision-making is used as a starting point for creating family of new decision-makings, which will formed with the approaches that used to solve the goal achievement problem.

It should be noted, that the system synthesis of decisionmakings is hard-formalized process in which a decisive role is played by expert-constructor. The effectiveness of this process depends on the quality of the performance of cognitive operations.

However, there are areas in which existing information technology can support and improve the performance of the process of system synthesis. This primarily concerns the areas of generation of sets of variants (using a combinatorial approach), evaluation and selection (using the knowledge base that reflects the preferences of the decision maker model or set of selection rules).

\section{REFERENCES}

[1] A.E. Kondratiev, "Network-centric front. Fighting in a single information space," in National Defense, vol. 2, 2011. (in russian)

[2] V. Dunayev, E. Medvedovsky, "Nuclear power plants automation and control systems," in Control Engineering Russia, vol. 3 (45), 2013. (in russian)

[3] V.N. Volkova, V.N. Kozlov, System Analysis and Decision Making: Reference Dictionary, Moscow: Higher. sch., 2004. 616 p. (in russian)

[4] D.E. Hank, A.J. Rights, D.U. Uichern, Business forecasting. 7th ed., Moscow: Publishing House "Williams," 2003. 656 p. (in russian)

[5] L.N. Butenko, D.V. Butenko, "The theory of systems. Objectives of conceptual design and their relationship to the laws of systems development," in Systems engineering, vol. 2, 2004. (in russian)

[6] D.P. Oleynikov, L.N. Butenko, Evaluation of the quality of objects and processes in the educational environment. Verbal decision analysis: a monograph, MISiS, VSTU, Moscow, Volgograd: 2006. 146 p.(in russian)

[7] D.P. Oleynikov, L.N. Butenko, "On the methodology of the system of decision-making methods of synthesis," Proceedings of the Congress on Intelligent Systems and Information Technology "IS \& IT'15", vol. 1, Taganrog: Publishing House of the SFU. pp. 160-165. (in russian)

[8] D.P. Oleynikov, L.N. Butenko, Synthesis methods of decision-making based on the principle of polarity // Successes of modern science: scientific-theoretical. Journal / Natural Sciences Academy, vol. 11, 2005. pp. 34-36. (in russian)

[9] D.P. Oleynikov, L.N. Butenko, S.P. Oleynikov, V.A. Kamaev, "Verbal analysis solutions. Synthesis method of hierarchical classification, " in Information technologies, vol. 6, 2007, pp. 29-37. (in russian)

[10] D.P. Oleynikov, L.N. Butenko, H.M. Moschkovich, A.I. Mechitov, "ARACE - A New Method for Verbal Decision Analysis," in International Journal of Information Technology \& Decision Making, vol. 15, № 1, 2015, pp. 115-140. DOI: 10.1142/S0219622014500801

[11] D.P. Oleynikov, L.N. Butenko, "Using a system of methods of synthesis methodology solutions to create a method of verbal analysis solutions ARACE," in Volga Research Bulletin, № 5-1 (45), 2015, pp. 99-105. (in russian)

[12] D.P. Oleynikov, L.N. Butenko, S.P. Oleynikov, Synthesis methods of decision-making on the basis of morphological approach // The current state of the natural sciences and engineering, № 18, 2015, pp. 87-91.(in russian)

[13] L.N. Butenko, S.P. Oleynikov, D.P. Oleynikov, "Decision support system Unicum," in Software products and systems, vol. 1, 2014, pp. 97-106. (in russian)

[14] D.P. Oleynikov, L.N. Butenko, Morphological based method and system for computer aided conceptual synthesis of decision-makings // Proceedings of the European Conference on Data Mining in 2014 and International Conferences on Intelligent Systems and Agents 2014 and Theory and Practice in Modern Computing 2014 - Part of the Multi Conference on Computer Science and Information Systems, MCCSIS 2014. pp. 117-124.

[15] D.P. Oleynikov, L.N. Butenko, "Automated system for the synthesis of decision-makings Alfa-Sintez," in Software products and systems, vol. 1, 2014, pp. 106-111. (in russian)

[16] D.P. Oleynikov, L.N. Butenko, Use the pattern "Inversion method of forming an alternative estimates" for the synthesis of the decisionmakings // The current state of the natural sciences and engineering, № 18, 2015, pp. 78-86.(in russia 